ISSN 1409-2441
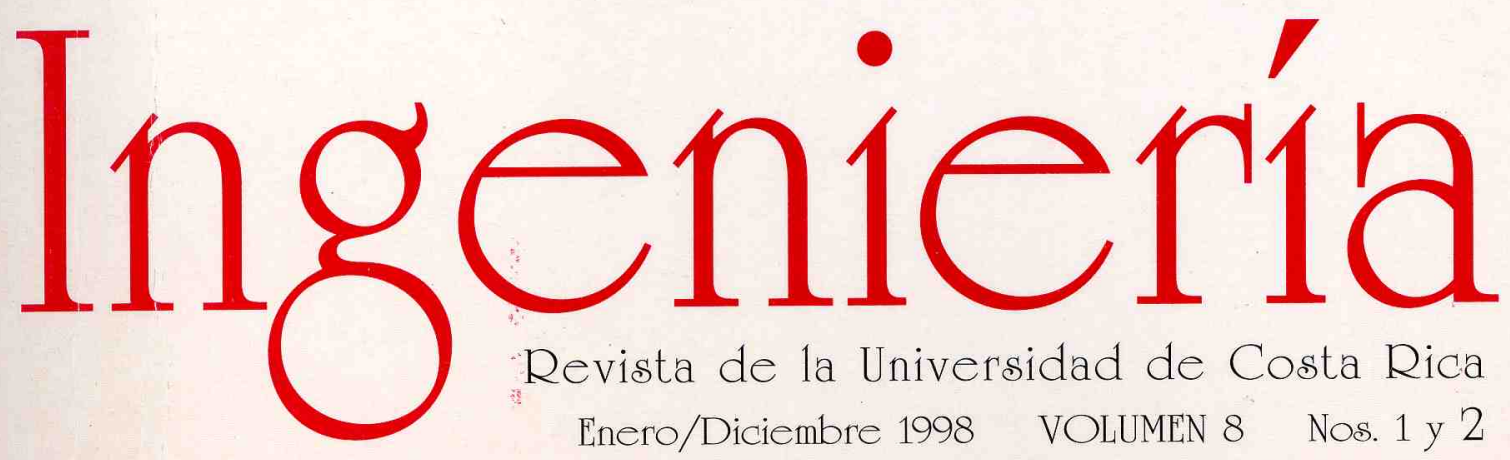

Revista de la Universidad de Costa Rica Enero/Diciembre 1998 VOLUMEN 8 Nos. 1 y 2 


\title{
POLINOMIOS CONCENTRADOS DOBLEM.ENTE ORTOGONALES EN EL DISEÑO DE FILTROS DIGITALES DE FASE LINEAL
}

Jorge A. Romero C.'

\begin{abstract}
Resumen
Los polinomios concentrados doblemente ortogonales, sintetizados a partir de la maximización de la energía en un intervalo pero en detrimento de la energía en otro, se utilizan en la aproximación por minimos cuadrados de la magnitud de filtros de respuesta linita al impulso de tase lineal. Junto con la técnica de la descomposición de valor singular, se emplean para calcular los coeficientes de la respue>ta al impulso, los cuales defmen tales filtros. Varios ejemplos ilustran lo aplicable del método propuesto; tal aplicabilidad se da tanto directamente (sin interferencia de la aproximación por minimos cuadrados) como mediante tal aproximación.
\end{abstract}

\section{Summary}

The dOllbly orthogonal concentrated polynomials, obtained afier maximizing an energy ratio in an interval at the expense of the tllergy in another, are used in the lea,t-squares approximation of the magnitude function of fmite impulse response digital filters with linear phase. A10ng with singular value decomposition, these polynomials are used to calculate the impulse response coefficients which dlaracterize tbe fihers. Several examples show their applicability. They can be applied both directly (WithOllt using aleas! -squares approximation) as well as by using the approximation.

Descriptores: Polinomios; funciones ortogonales; optimización; tratamiento y detección de sefíales.

\section{INTRODUCCIÓN}

Eldiseliode filtros digitales de fase lineal

El diseíio de filtros digitales es un tema recurrente dentro de la literatura técnica. La búsqueda de algoritmos y enfoques que garanticen un diselio óptimo bajo un criterio determinado, constituye un esfuerzo común dentrode la investigación en esta área.

Losfiltros digitales se clasifican en dos tipos, los filtros de respuesta finita al impulso (que denominaremosFIR de acuerdo con sus siglas en inglés) y los filtros de respuesta infinita al impulso(IIR). Si se compararan ambos tipos de filtros para efectos de uso, lo usual es hacerlo sobre la base del níunero de multiplicaciones por muestra, requerido en el métodoestándar de realización para cada tipo defiltro. Siguiendo tal criterio, para el diselio de filtros pasobajo con características de rizado casi uniforme, los filtros elípticos IIR se construyen más eficientemente que los filtros FIR de fase lineal equivalentes. Al agregársele a las especificaciones de diselio el requerimiento adicional del retardo de grupo constante en la banda de paso, los filtros FIR de fase lineal presentan una construcción más eficiente que los filtros I1R elípticos con retardo de grupo ecualizado [1].

La comparación sobre la base del número de multiplicaciones por muestra se justifica puesto que, desde los puntos de vista de programación y de construcción, tal número es una excelente medida de la complejidad del diselio así como es también el factor determinante de la razón máxima de procesamiento del sistema. Sin embargo, hay otros métodos para comparar los dos tipos de filtros, en particular, cuando se consideran los 
diversos efectos debidos a la longitud de palabra finita que ocurren en un diselio práctico.

Entre tales efectos se tiene el ruido de redondeo, tanto el correlacionado como el no correlacionado y la sensibilidad a la cuantificación de los coeficientes. Para la construcción de un filtro FIR en forma directa. el ruido de redondeo máximo se reduce acumulando sumas parciales en un registro de longitud extendida y luego redondeando el resultado final. Para los filtros IIR construidos en cascada en aritmética de punto fijo, el ruido de redondeo está relacionado con el problema del ámbito dinámico: la varianza del ruido no correlacionado de redondeo puede entonces minimizarse usando las técnicas del apareamiento de polos y ceros y el ordenamiento de las secciones del filtro [2J. El ruido correlacionado de redondeo puede eliminarse tanto usando esquemas apropiados de cuantificación en determinadas estructuras como incorporando mecanismos de saturación adecuados en dispositivos aritméticos [31.

Para resolver el problema de cuantificación de los coeficientes, se suele escoger longitudes de palabra diferentes para los valores de los coeficientes y los valores de la selial [31. La longitud de palabra de los coeficientes se escoge para satisfacer especificaciones de respuesta en frecuencia. en tanto que la longitud de palabra de la selial se escoge para satisfacer una razón de señal a mido determinada.

Desde un punto de vista cuantitativo, es más fácil comparar los dos tipos de filtro utilizando como medida el número de multiplicaciones por muestra. Considerando además que los filtros (ya sea continuos o digitales) de fase lineal, son preferidos a los filtros con fase arbitraria debido a su retardo de grupo constante. no es de extrañar que los filtros digitales con fase lineal hayan recibido tanta atención en la literatura.

De los dos tipos de filtro considerados. los filtros FIR son los que de una forma más simple pueden manipularse para obtener una fase lineal, permitiendo así que el diseño se concentre en la característica de magnitud que el filtro va a poseer [2-4]. El principal obstáculo que enfrentan los filtros IIR para que sean compelidos a poseer fase lineal es el hecho de que. para un filtro discreto causal. lineal e invariante con el tiempo de fase mínima, tanto su fase como su magnitud se relacionan por una transformada de Hilbert [5]. lo que implica que al especificar independientemente las características de fase de las de magnitud. la relación mencionada se vería afectada con toda probabilidad [6].

Aparte de ser fácilmente compelidos a tener exactamente fase lineal. los filtros FIR no presentan problemas de estabilidad. Se han empleado diferentes técnicas para diseíiarlos y pueden clasificarse en: (1) método de ventanas. (2) muestreo en frecuencia y (3) diseños de rizado uniforme [7].

Una ventana es una función de peso limitada en el tiempo utilizada para controlar la convergencia de una serie de Fourier mediante la modificación de los respectivos coeficientes. Un filtro FIR que aproxime una determinada respuesta en frecuencia necesita del truncamiento de la serie de Fourier. lo que da origen al fenómeno de Gibbs [8]. El efecto de este fenómeno se aminora multiplicando la ventana por los coeficientes de la serie tmncada. El diseño por ventanas es una técnica bien establecida $[9,101$.

El segundo método para aproximar un filtro con especificaciones de respuesta en frecuencia dadas es mediante el muestreo de la respuesta deseada en $\mathrm{N}$ puntos. donde $\mathrm{N}$ es el número de muestras en la respuesta al impulso del filtro. El muestreo puede ser uniforme o no uniforme. Para las bandas de transición. deben escogerse cuidadosamente los valores de la respuesta en frecuencia para optimizar el diselio del filtro (11-16).

La tercera técnica para diseliar filtros FIR involucra la solución de un sistema de ecuaciones no lineales para generar un filtro con un error de aproximación de rizado 
uniforme. Las cantidades desconocidas son tanto los coeficientes de la respuesta al impulso como el conjunto de frecuencias donde ocurren los valores extremos del error de aproximación. Las ecuaciones no lineales se escriben como restricciones sobre tales valores extremos y sobre las derivadas. Técnicas estándares de optimización no lineal se usan luego para resolver las ecuaciones [1722].

Una técnica reciente basada en la mezcla de los dos métodos anteriores consiste en utilizar polinomios ciclotómicos para formar un prefiltro sin multiplicadores, para luego adicionarJe un ecualizador. Se propone una estrategia para escoger los polinomios adecuados para satisfacer especificaciones del filtro, en tanto que el ecualizador se puede diseílar de dos formas: un ecualizador basado en muestras espaciadas uniformemente construido, óptimamente, mediante un algoritmo 1\}lodificado de Parks-McClelJan [21], o un ecualizador basado en muestras espaciadas irregularmente, construido empleando métodos de selección de

$$
R(f)=\frac{\int_{I_{a}}|f(x)|^{2} d x}{\int_{I_{b}}|f(x)|^{2} d x}
$$

donde $l a, \mathbf{h}$ son intervalos de la recta numérica. ReO puede interpretarse como una razón de energía. Al polinomio $f o(n)(x)$ que maximiza tal razón se le puede ver como el polinomio con la energía más concentrada en el intervalo $l a$ a expensas de su energía en $\mathrm{h}$.

El problema de maximizar ReO es equivalente al de encontrar el autovalor más grande $A / n$ ) al que corresponde la autofunción $\left.j^{\prime}\right)(x)$ dentro de un problema de autovalores. Las autofunciones restantes, polinomios de orden subcolljuntos de funciones base complejas. Esta técnica reduce el número de adiciones y multiplicaciones requeridas a costa de agregar un pequelio porcentaje de retardos adicionales. Reduce también la sensibilidad a la cuantificación de los coeficientes como al mido de redondeo $[23 \mathrm{~J}$. Se ha propuesto asimismo usar un tipo especial de programación lineal para el diseílo basado en polinomios ciclotómicos [24].

En este artículo se propone usar el método de muestreo uniforme en frecuencia para diseliar filtros FIR de fase lineal utilizando polinomios concentrados doblemente ortogonales. Se propone usar estos polinomios tanto directamente como por medio de la solución a un problema de aproximación de mínimos cuadrados.

\section{Polinomios concentrados doblemente ortogonales}

Los polinomios concentrados doblemente ortogonales $(n)(x)$ son polinomios de orden $n$ surgidos al maximizar la razón

$n$, igualmente integran junto con $\left.i j^{\prime \prime}\right)(x)$. un conjunto de funciones $\operatorname{fr}(x), j=0, \ldots, 11$ ortogonales simultáneamente sobre la e $l b$ [25]. Un problema similar fue resuelto involucrando en la solución un conjunto completo de funciones ortogonales de banda limitada tanto sobre un intervalo finito como sobre toda la recta numérica [26].

Sea $1 / J O(x), \quad / / J_{i}(x), \quad . H . \quad t j J_{,},(x)$ una base para el espacio $F_{n}$ de polinomios de orden 11. Cada polinomio en Fn se describe como:

$$
f(x)=\underset{k}{i f k 9 k} \quad(x)
$$


Por lo que se le puede representar vectorialmente como $\mathbf{1}=[f o, \mathrm{Jl}, \quad$ "',, $\boldsymbol{f}$, , (Con esta última notación, $R(f)$ puede escribirse como:

$$
R(f)=\frac{f^{T} A \underline{f}}{\underline{f}^{T} B \underline{f}}
$$

$\boldsymbol{f}$ es la transpuesta de $1, A$ y $B$ son matrices $(n+1) \times(n+1)$ con elementos descritos por:

$$
\int \phi_{i}(x) \phi_{j}(x) d x
$$

que son integrales evaluadas sobre $l a$ e $\boldsymbol{h}$, respectivamente. Para maximizar $R(f)$ se resuelve la ecuación matricial de autovalores

$$
A f=W f
$$

El mayor autovalor / que satisface la ecuación anterior da la razón máxima para $R(f), l / n)$. Las coordenadas .k del autovector correspondiente son los coeficientes del polinomio en la ecuación 2 que hace $R(f)=$ $l / n)$, fin) $(x)$.
Una base conveniente que puede usarse para calcular los polinomiosfn) $(x)$ es $t A(X)=x i$. Si $l a$ $=[a l, \quad a 2], \quad \boldsymbol{h}=\left[b_{1}, b_{2}\right]$, puede demostrarse que las entradas de las matrices $A=$ [ay $] \mathrm{y}$ $B=[b y]$ están dadas por:

$$
a_{i j}=\frac{a_{2}^{i+j+1}-a_{1}^{i+j+1}}{i+j+l} \quad, \quad b_{i j}=\frac{b_{2}^{i+j+1}-b_{1}^{i+j+1}}{i+j+l}
$$

Cualquier otra base puede utilizarse para realizar el cálculo de los polinomios que maximizan $R(!)$. La naturaleza de tales polinomios depende de si $l a \circ h$ son disjuntos o no. Si lo son, se habla de un problema exterior. Si la e $\mathrm{h}$, se habla de un problema interior [25].

Ejemplo $\mathrm{N}^{\circ} 1$ Para el caso definido por $\boldsymbol{h}=[-$ $0.5,0.5], \quad l a=[-0.1,0.1] Y n=5$, se muestra en la Figura $\sim l$ los seis polinomios $f n)(x)$ que maximizan la razón de energía estudiada. Esto corresponde a un problema interior.

EjemploN 2 Un problema interior asimétrico se ilustra con $h=[-0.5,0.5]$, la $=[0.05,0.35] \quad \mathrm{Y}$ $n=5$. La Figura 2 muestra los resultados obtenidos empleando la base propuesta en el texto.

\section{APROXIMACIÓN DE MÍNIMOS CUADRADOS USANDO POLINOMIOS CONCENTRADOS DOBLEMENTE ORTOGONALES}

La doble ortogonalidad característica de los polinomios $f n)(x)$ es útil en ciertos problemas de aproximación por minimos cuadrados.

Las dos ecuaciones de ortogonalidad para los polinomios $f n)(x)$ son:

$$
\begin{array}{ll}
f_{l b} f(n)(x) f k(n)(x) d x & =8 l^{l k} \\
f_{f / n)(X) ! k(n)(x) d x} & ={ }_{1 ! j}^{1(n) s:} \quad \text { u;k }
\end{array}
$$

Para), $k=\mathrm{O}, 1, \ldots, n$ y para $n=0,1,2, \ldots$. Supóngase que se busca un polinomio de grado $n J(x), \quad$ para aproximar una función dada $g(x)$ y que el error de aproximación se define como: 
$E=O J_{a} f_{I a} /(x)-g(x) 1^{2} d x+o J_{b} f_{l b} I /(x)-g(x) 1^{2} d x$

donde las constantes wa y (ob son pesos positivos reales. Para minimizar E, escríbase $f(x)$ como:

$\left.j(x) \quad=L_{j=0}^{n} c j f / n\right)(x)$

Considerando la soluciónf(x) así como la función a aproximar $g(x)$ como entidades reales, se tiene

$E=\operatorname{OJ~} a_{l a} f\left(/_{(X)}-g(X »)_{2} d x+0{ }_{l a} b \underset{l b}{f} / /_{(X)}-g(X »)_{2} d x\right.$

Derivando con respecto a $C_{j}$ e igualando a cero

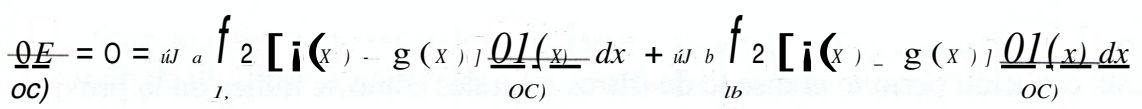

Nótese que

$\frac{\partial f(x)}{\partial c_{j}}=\frac{\partial}{\partial c_{j}} \sum_{j=0}^{n} c_{j} f_{j}^{(n)}(x)=f_{j}^{(n)}(x)$

Sustituyendo en la penúltima ecuación,

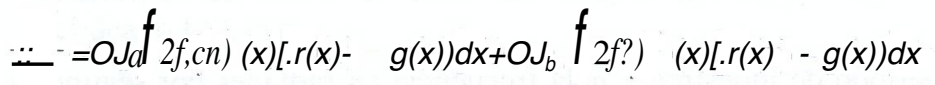

$0=\mathrm{rO}_{a} f_{2 f t}^{l a} \quad$ (X) $f(x) d x \quad$ - úJ $\left.f_{a}^{l b} 2 f t\right) \quad(x) g \quad(x) d x$

$+{ }^{{ }^{1 a}}{ }_{b} f_{2}$ /?) $(x) \quad /(x) d x \quad-{ }_{\text {(e) } b}^{1_{a}} f_{2}$ It $) \quad(x) g \quad(x) d x$

Haciendo uso de las ecuaciones de ortogonalidad,

$\left.\left.f f l n)(X) f(X) d x=f_{l a} f\right) \quad n\right)(X) \underset{m=O}{\dot{\ell}_{l a}}$ e $\left.m f \sim n\right)(X) d x$

$$
\begin{aligned}
& =c_{j} \lambda_{j}^{(n)} \quad \dot{\mathbf{S I}]}=m \\
& =0 \quad \text { si\} }\} \cdot f \cdot . \cdot-m
\end{aligned}
$$


$\left.\left.f_{l b} f t\right) \quad(x) f(x) d x \quad=f_{l b} f l^{\prime \prime}\right)(X) \sum_{m=O}$ e $\left.m j \sim ; "\right)(x) d x$

$$
\begin{aligned}
& =C_{f} \quad s i j=m \\
& =0 \quad \text { sij }-: t:-m
\end{aligned}
$$

Finalmente.

$$
0=\left\{U a C, A\left(t-{ }_{(U a} f_{f i n)}(x) g(x) d x+\{u b e\lrcorner-\left(U b f_{f i n)} \quad(x) g(x) d x\right.\right.\right.
$$

Los valores de e $i$ que resuelven el problema de aproximación por mínimos cuadrados son entonces,

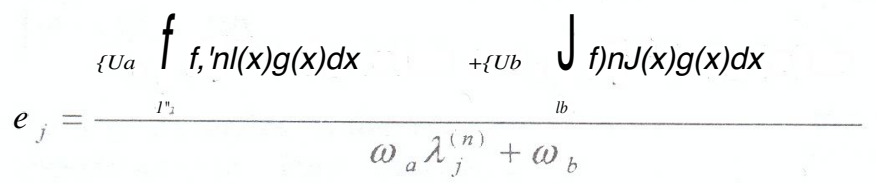

con $\}=$ O. 1. 2.... 11. Esta ecuación permite el disei'i.o de filtros digitales como se indica en la próxima sección.

\section{APLICACIONES EN EL DISEÑO DE FILTROS DIGITALES}

La respuesta en frecuencia de un filtro digital se define por

$$
H\left(e . /^{W T}\right)=i_{11=0}^{i t f} \operatorname{Lh}(n) e-J W n T \quad-=H(r u)
$$

$h(n)$ es la respuesta al impulso. $T$ el periodo de muestreo y $(v$ la frecuencia en radianes por segundo. Para el diselio de un filtro digital de fase lineal, la respuesta en frecuencia se hace simétrica o antisimétrica alrededor de un punto medio y en cada caso. Al puede ser impar o par [31. Para un diselio simétrico con Al par.

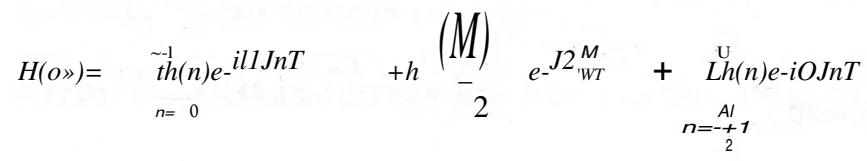

lo que después de alguna álgebra y usando simetría. cambia a [4]

$$
H(\omega)=e^{-j \omega \frac{M}{2} T}\left\{h\left(\frac{M}{2}\right)+2 \sum_{i=0}^{\frac{M}{2}-1} h(i) \cos \left[\left(\frac{M}{2}-1\right) \omega T\right]\right\}
$$

Para un diseílo simétrico con $\mathrm{Al}$ impar [4J 


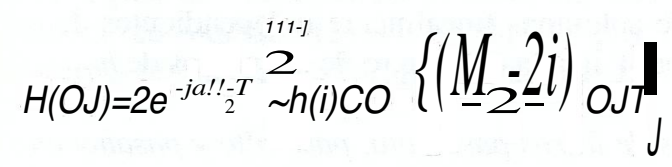

Las dos ecuaciones anteriores tienen la forma

$$
H\left((0)=A\left((0) e \quad-j^{\prime \prime}, M{ }_{2} T\right.\right.
$$

donde $A(O J)$ es una función real de la variable $O J$ y queda e:'])resada en términos de la respuesta al impulso $h(n)$. La ecuación 14 indica claramente que se puede aproximar $A(O J)$ mediante tilla expansión polinomial semejante a la dada en la ecuación 8. Si tal es el caso, se tratará de una aproximación de mínimos cuadrados.

Aplicación directa de los polinomios $\operatorname{In})(x)$ al diseño defiltros digitales

Por aplicación directa se entenderá el empleo de uno de los miembros.on)(x), para algún $n$ y algún\}, en el diselio de un filtro digital. Para ello, se establece la siguiente ecuación

$$
\text { J) } \left.)_{(u)}\right)=h\left(\simeq-J+2 \underline{\underline{\Psi}} h(i) \cos \left[\left(\underline{\mathbb{N}}-\mathbf{i} J_{u J} T\right]\right.\right.
$$

En total, hay $M I 2+1$ incógnitas. Extendiendo la ecuación a $L$ frecuencias $\{0 J\}$, se establece un sistema sobredeterminado de ecuaciones que se puede resolver usando la descomposición de valor singular (SVD), que proporciona una solución $h(n)$, óptima bajo el criterio de mínimos cuadrados lineales. Los coeficientes del filtro, es decir, los valores del vector $h(n)$, tendrán norma mínima en el sentido euclideano [27)8J.

El número $L$ de frecuencias debe ser mayor o igual que $M I 2+1$. El sistema de ecuaciones mencionado en el párrafo anterior se escribe como $B h .=$ fí, donde $h$. es el vector de coeficientes del filtro, fí es el vector de valores resultante al evaluar el polinomio en las frecuencias $\{0 J\} \mathrm{Y} B$ es la matriz originada al evaluar la función coseno en la ecuación 15. B tiene entradas de la forma

$2 \cos \left[\left(\frac{M}{2}-i\right) \omega_{j} T\right]$

donde $\}=1, \ldots, L$ e $i=\mathrm{O}, \ldots, \mathrm{Ml} 2-1$. Una de las columnas o filas de $B$ (dependiendo del ordenamiento escogido) estará constituida por solamente unos. La solución del sistema se obtiene en términos de los vectores singulares derechos !:: y los valores singulares $\left\{0^{\prime}\right\}$ provenientes de la descomposición de valor singular de $B$ :

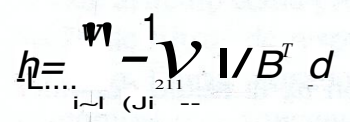


donde $W$ es el rango de $B$, definido como el número de columnas linealmente independientes de la matriz. En nuestro caso, la solución es consistente porque $W$ iguala el número de elementos de b...

Ejemplo $\mathrm{N}^{\circ} 3$ Las figuras JV2 3, 4 Y 5 muestran ejemplos defiltros paso bajo, paso alto y pasabanda, respectivamente, disePíados de acuerdo con el procedimiento indicado. Para variar parámetros de frecuencia de corte, banda de paso y de rechazo, atenuación y otros que son usuales en la teoría de filtros, se modifica el orden $n$ de los polinomios $j n)(x)$ junto con los intervalos de ortogonalidad la eh El orden $n$ incide directamente sobre la atenuación, en tanto que la especificación de los intervalos lo hace sobre los parámetros defrecuencia de corte y las bandas de paso y de rechazo.

Combinación del criterio de mínimos cuadrados y polinomiosjn) (x) para el disePío de filtros digitales

Suponiendo que $l a=[a l, a 2], h=l b_{i}$, b2] Y que la está contenido dentro de $l b$ (problema interior), la solución al problema de mínimos cuadrados se escribe como:

$$
\begin{aligned}
c_{j}= & \frac{1}{\omega_{a} \lambda_{j}^{(n)}+\omega_{b}}\left\{\left(\omega_{a}+\omega_{b}\right) \int_{a_{1}}^{a_{2}} f_{j}^{(n)}(x) g(x) d x+\omega_{b}\left[\int_{b_{1}}^{a_{1}} f_{j}^{(n)}(x) g(x) d x\right.\right. \\
& \left.\left.+\int_{a_{2}}^{b_{2}} f_{j}^{(n)}(x) g(x) d x\right]\right\}
\end{aligned}
$$

La anterior ecuación se aplica en el diselio de un filtro digital estableciendo la igualdad

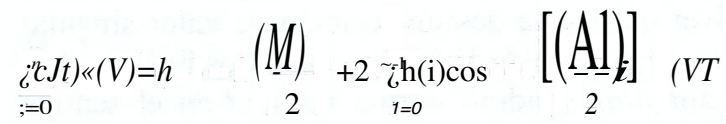

En la derivación de la ecuación 17, se supuso que no hay una región de transición entre las bandas de paso y de rechazo del filtro, por lo que fácilmente se puede identificar la banda de paso con la Y la banda de rechazo con lo que resta de $l b$. Para proceder con el diselio, se sigue el procedimiento esbozado en la sección anterior. Dentro de la banda de rechazo, $g(x)$ toma un valor pequelio, en tanto que para la banda de paso, $g(x)=1$.

Ejemplo $\mathrm{N}^{\circ} 4$ En la Figura $N^{\circ} 6$ se presenta un disePío de filtro paso bajo donde la región de transición entre bandas es nula, es decir, se aproxima un filtro paso bajo ideal. Para la banda de rechazo, $g(x)=0.001$.

Para mejorar la respuesta de magnitud del diselio anterior, la región de transición debe suponerse no nula para efectos de disminuir el rizado de aproximación; es aconsejable que se defina entonces una función continua que realice una transición suave entre bandas [29.30]. A modo de ejemplo. considérese el pulso de pendiente senoidal mostrado en la Figura $\mathrm{N}^{\circ}-7$. de uso en telecomunicaciones para eliminar la interferencia entre símbolos [31). La transición del valor 1 al valor $\mathrm{O}$ del pulso se realiza por medio de la función

$$
g_{t}(\Delta \omega)=\frac{1}{2}\left[1-\operatorname{sen}\left(\frac{\pi \Delta \omega}{2 \omega_{x}}\right)\right]
$$

donde $\left./ \mathrm{j},\left\{_{\mathrm{i}}\right)={ }_{\mathrm{i}}\right)-\left\{_{i}\right)$, con $(\sim$ la mitad del valor de la longitud de la región de transición y (Veel valor central de tal región. 
Si la región de transición no es nula, hay dos casos a considerar: (1) la banda de paso conforma el intervalo de ortogonalidad $l a \mathrm{Y}(2)$ tanto la banda de paso y la región de transición conforman tal intervalo.

Para el primer caso anterior, el cálculo de los coeficientes $C j$ se modifica como:

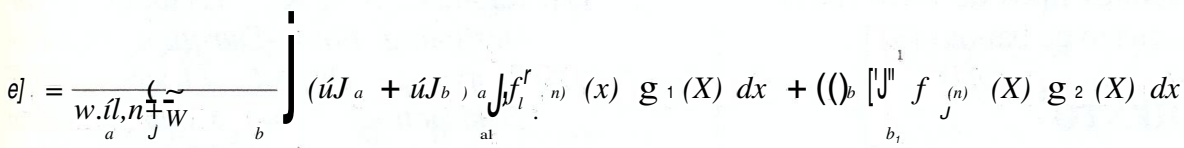

$$
\begin{aligned}
& \left.\left.\left.+\left\{f^{\prime \prime \prime}(x) g, \quad(x) d x+1, f\right)^{\prime \prime \prime)}(x) g, \quad(x) d x+1, f\right\}^{\prime \prime \prime} \quad(x) g, \quad(x) d x\right]\right\}
\end{aligned}
$$

Los intervalos [C1, ai] Y [a: C2] forman la región de transición en la que se define una función $g_{i}\{x$ ), que define la transición del valor 1 al valor $\mathrm{O}$ del pulso con pendiente senoidal mostrado en la Figura N2 7. $\mathrm{gl}(x)$ vale 1 en tanto que $g 2(X)$ será un número cercano a $\mathrm{O}$.

Ejemplo N 5 En la Figura IV'- 8 se muestra un filtro paso bajo diseñado con los parámetros siguientes: ,ú); $=1, u ́ J_{a}=0.001, \mathrm{gl}(x)=1, g 2(x)$ $=0.00001, \quad l a=[-0.2,0.2], \quad \mathrm{h}=[-0.5 .0 .5], \quad \mathrm{c} 2=$
$0.3153 \quad Y \mathrm{C} 1=-0.3153$. El orden de los polinomios utilizados es $n=30$, un orden menor que los del ejemplo inmediato anterior.

En el segundo caso mencionado, cuando la región de transición y la banda de paso integran el primer intervalo de ortogonalidad la y siguiendo la notación utilizada. se tiene que $l a=\left[\begin{array}{ll}C 1, & C 2\end{array}\right]$. Los coeficientes $c_{j}$ se calculan ahora como:

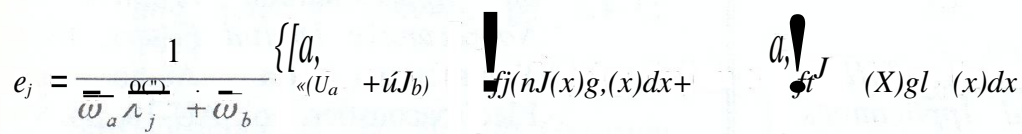

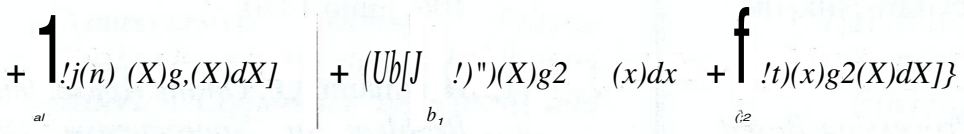

Ejemplo $\mathrm{N}^{\circ} 6$ En la Figura IV'- 9 se muestra un filtro paso bajo diseñado con los parámetros

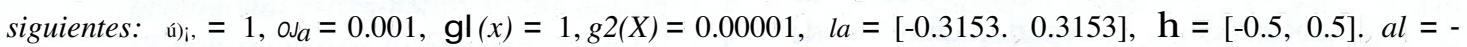
$0.2 \mathrm{Ya} 2=0.2$. El orden de los polinomios utilizados es $n=30$.

Otras funciones pueden sugerirse para trazar la región de transición entre las bandas de paso y de rechazo [30]. La usada aquí constituye un ejemplo.

\section{CONCLUSIONES}

En este artículo se ha presentado un método de diselio de filtros de respuesta finita al impulso con fase lineal, basado en la aplicación de polinomios concentrados doblemente ortogonales. Se utilizó el criterio de mínimos cuadrados para realizar una aproximación y, posteriormente, se utilizó la descomposición de valor singular para encontrar los coeficientes de la respuesta al impulso que definen el filtro digital. 
Aunque los ejemplos presentados corresponden a diselios para un filtro paso bajo en su mayoria, mediante las transfonnaciones espectrales adecuadas, tales diselios pueden adecuarse a los restantes tipos de filtro (paso alto, pasabanda y rechazo de banda) [32].

\section{AGRADECIMIENTO}

Al ingeniero José Miguel Páez Jiménez, en su momento Director de la Escuela de Ingeniería Eléctrica, por su apoyo para la inscripción del proyecto de investigación que dio como resultado este artículo.

\section{BIBLIOGRAFÍA}

[1] Rabiner, L. R, el. al., Some Comparisons between FIR and JIR Digital Filters, Bell System Technical. Journal, vol. 53, pp. 305-331, febrero 1974.

[2] Jackson, L. B. Digital Filters and Signal Processing. Tercera edición. Kluwer Academic Publishers: Boston, 1996.

[3] Antoniou, A. Digital Filters: Ana ysis, Design and Applications. Segunda edición. McGraw-Hill, Inc.: New York, 1993.

[4] Romero, I, Signal Processing Based on Irregular Sampling: Reconstruction, Compression, and Signal Transformation. Tesis de doctorado. Departamento de Ingeniería Eléctrica y de Computación, Concordia University, Montréal, Québec, Canadá, junio 1996.

[5] Papoulis, A. The Fourier Integral and Its Applications. McGraw-Hill Publishing Company: New York, 1987.
[6] Zadeh, L. A. el. al. Linear System Theory. McGraw-Hill Book Company, Inc.: New York, 1963.

[7] Rabiner, L. R., Techniques for Designing Finite-Duration ImpulseResponse Digital Filters, IEEE Transactions on Communication Technology, vol. COM-19, pp. 188 . 195, abril 1971.

[8] Lathi, B. P. Communication . ystems. John Wiley \& Sons, Inc.: New York, 1968.

[9] Bose, N. K. Digital Filters: Theory and Applications. Elsevier Science Publishing Co., Inc.: New York, 1985.

[10] Adams, I W., A New Optimal Window, IEEE Transactiolls 011 Signal Processing, vol. 39, no. 8, pp. 1753-1769, agosto 1991.

[11] Rabiner, L. R, el. al., An Approach to the Approximation Problem for Nonrecursive Digital Filters, IEEE Transactions on Audio and Electroacoustics, vol. AU-18, pp. 83106, junio 1970.

[12] Hernnann, O., On the Approximatioll Problem in Nonrecursive Digital Filter Design, IEEE Transactions on Circuit Theory, vol. CT-18, pp. 411413, mayo 1971.

[13] Algazi, V. $\mathrm{R}$ et. al., On the Frequency Weighted Least-Square Design of Finite Duration Filters, IEEE Transactions on Circuits and Systems, vol. CAS-22, no. 12, pp. 943-953, diciembre 1975.

[14] Bozic, S. M. el. al., FIR design by non-uniform sampling in frequency domain, International Journal of Electronics, vol. 65, no. 4, pp. 815821, 1988. 
[15]Summers, B, el. al., $n R$ Digital Filfer Design Using Non-Equispaced Frequency Sampling. Electronics Letters. vol. 25, no. 5, pp. 338-339, marzo 1989.

[16]Kim. 1. T.. el. al., Design of Nonuniformly Spaced Linear-Phase FIR Filters Using lvlixed Integer Linear Programming. IEEE Transactions on Signal Processing, vol. 44, no. 1, pp. 123-126, enero 1996.

[17]Herrmann, O., "Design of Nonrecursive Digital Filters with Linear Phase". Electronics Letters, vol. 6. no. 11, pp. 328-329, mayo 1970.

[18]Hofstetter. E., et. al., A New Technique for the Design of NonRecursive Digital Filfers, Proceedings of the 5th. Annual Princeton Conference on Information and Science Systems, pp. 64-72, 1971.

[19]Hofstetter, E., el. al., On Optimum Nonrecursive Digital Filters, Proceedings of the 9th Annual Allerton Conference on Circuit and , ystem Theory, pp. 789-798, 1971.

[20]Holt, A. G. 1., el. al. The use of orthogonal polynomials in the design of non-recursive linear phase digital jilters, International Journal of Electronics, vol. 38, no. 3, pp. 385392, 1975.

[21]Rabiner, L. R., el. al., $n R$ Digital Filter Design Techniques Using Weighted Chebyshev Approximation, Proceedings of the IEEE, vol. 63, pp. 595-610, abril 1975.

[22] Tufts, D. W., Comments on $n R$ Digital Filfer Design Techniques
Using Weighted Chebyshev Approximation, Proceedings of the IEEE, pp. 1618-1619, noviembre 1975.

[23]Hartnett, R. 1. el. al., On the Use of C'yclotomic Pofcvnomial Prejilters for Efficient $n R$ Filter Design, IEEE Transactions on Signal Processing, vol. 41. no. 5, mayo 1993.

[24]Oh, W. 1., el. al., "Design of Efficient FIR Filters with Cyclotomic Polynomial Prefilters Using Mixed Integer Linear Programming", IEEE Signal Processing Letters, vol. 3, no. 8, pp. 239-241, agosto 1996.

[25] Gilbert, E. N., et. al., "Doubly Orthogonal Concentrated Polynomials", SIAM Journal on Mathematical Analysis, vol. 4, no. 2, pp. 290-319, abril 1977.

[26] Slepian, D. et. al., "Prolate Spheroidal Wave Functions, Fourier Analysis and Uncertainty-I", Bell System Technical Journal, vol. 40, pp. 43-63, enero 1961.

[27]Haykin, S. Adaptive Filter Theory. Segunda edición. Prentice-Hall, Inc.: Englewood Cliffs, 1991.

[28]Dowling, E. M., el. al., 'The Equivalence of the Total Least Squares and MinimUlll Norm Methods", IEEE Transactions on Signal Processing, vol. 39, no. 8, agosto 1991.

[29] Gopinath, R. A., "Thoughts on Least Squared-Error Optimal Windows", IEEE Transactions on Signal

[30] Processing, vol. 44, pp. 984-987, abril 1996.

[31]Burrus, C. S., et. al., "Least Squared Error FIR Filter Design with 
Transition Bands". IEEE

Transactions on Signal Processing. vol. 40. pp. 1327-1340, junio 1992.

[32] Schwartz. M. Intormalioll Transmissioll, A\{odulafion and Noise. Tercera edición. McGraw-Hill Book Company: New York, 1980.

[33] Constantinides. A. G.. "Spectral Transfonnations for Digital Filters". Proceedings of the Institute of Electronics Engineers. vol. 117. pp. 1585-1590, agosto 1970. 